\title{
ON THE TOPOLOGY OF A COMPACT INVERSE CLIFFORD SEMIGROUP
}

\author{
BY
}

\author{
D. P. YEAGER
}

\begin{abstract}
A description of the topology of a compact inverse Clifford semigroup $S$ is given in terms of the topologies of its subgroups and that of the semilattice $X$ of idempotents. It is further shown that the category of compact inverse Clifford semigroups is equivalent to a full subcategory of the category whose objects are inverse limit preserving functors $F: X \rightarrow G$, where $X$ is a compact semilattice and $G$ is the category of compact groups and continuous homomorphisms, and where a morphism from $F: X \rightarrow G$ to $G: Y \rightarrow G$ is a pair $(\epsilon, w)$ such that $\epsilon$ is a continuous homomorphism of $X$ into $Y$ and $w$ is a natural transformation from $F$ to $G \epsilon$. Simpler descriptions of the topology of $S$ are given in case the topology of $X$ is first countable and in case the bonding maps between the maximal subgroups of $S$ are open mappings.
\end{abstract}

A popular topic of study in compact semigroups has been the question, for a given compact Hausdorff space, how many nonisomorphic continuous, associative multiplications of a given type will it admit? There is an older companion question, and that is, for a given algebraic structure, is there a compact Hausdorff topology which is compatible with all the operations, and if so, how many such topologies exist? It is known that the abelian groups which admit such a compact Hausdorff topology are certain products of copies of the group of rational numbers, $p$-adic groups, finite groups, and $Z\left(p^{\infty}\right)$ [4, Theorem 25.25]. But an abelian group may admit several such topologies. For example, the additive group of real numbers admits a compact $n$-dimensional topology for each positive integer $n$. In the nonabelian case, there is the 1932 result by van der Waerden [9], in which he described a system of "neighborhoods" about the identity of any group, which was finer than any compact group topology for which the identity was not isolated in the set of noncentral elements. He further proved that if a group admitted a topology giving it the structure of a compact simple Lie group, then each of these algebraically defined neighborhoods was a neighborhood of the identity relative to the given topology. Thus he gave what amounted to an algebraic description of the topology of a compact simple Lie group, which had an immediate

Received by the editors November $1,1974$.

AMS (MOS) subject classifications (1970). Primary $22 \mathrm{~A} 15$.

Key words and phrases. Compact semilattice, compact inverse Clifford semigroup, inverse-limit-preserving functor. 
generalization to semisimple Lie groups. It followed that an algebraic group admitting the structure of a compact semisimple Lie group must admit exactly one compact topology.

A similar result was achieved by J. D. Lawson in [6], in which he described a "convergence" for nets in an algebraic lattice, which enabled him to algebraically describe the open subsets of a compact lattice; he then used that result to give an algebraic method for determining the topology of a compact semilattice.

Given these results, one natural question to ask is, what may we say about those semigroups $S$ which are semilattices of groups in the strongest sense, i.e., inverse Clifford semigroups? The work of Bowman in [1] indicated that one might hope to describe the topology of $S$ in terms of those of the subgroups and the semilattice of idempotents of $S$. Such a description is achieved by Theorem 1 of this paper. Thus, in view of Lawson's result we can say that given an inverse Clifford semigroup $S$ with a preassigned compact topology on each of its subgroups, there exists at most one compact semigroup topology on $S$ which induces the preassigned topologies on the subgroups of $S$. We obtain this result as a corollary to Theorem 4 and to Corollary 16 of [6]. In $\$ 3$ we give a description of the category $\mathrm{Cl}$ of compact inverse Clifford semigroups which follows closely the outline of Bowman's description of the full subcategory of $C \ell$ whose objects $S$ satisfy the property that $E(S)$ (the semilattice of idempotents of $S$ ) is a perfect semilattice. Theorems 6 and 7 give simpler topological constructions for certain objects of the category $\mathrm{Cl}$.

\section{Introduction.}

Definition. A semilattice is a commutative, idempotent semigroup. In any semilattice $X$ there is a natural ordering $\leqslant$, defined by $x \leqslant y$ if and only if $x y=x$. It should be noted that for any two elements $x$ and $y$ of a semilattice $X$, the product $x y$ is the infimum relative to $\leqslant$ of the set $\{x, y\}$.

REMARK. We shall use the convention throughout this paper whereby a semilattice $X$ is considered a small category whose objects are the elements of $X$ and for which a morphism $x \rightarrow y$ exists from a point $x$ of $X$ to a point $y$ of $X$ if and only if $y \leqslant x$. If $F: X \rightarrow K$ is a functor from $X$ into any category $K$, then we denote $F(x \rightarrow y)$ by $F_{y}^{x}$.

Notation. Categories with which we shall be working in this paper are the category $C$ of compact Hausdorff spaces and continuous maps, the category CS of compact semigroups and continuous homomorphisms, and the categories $\Omega, G$, and $C l$ of compact semilattices, groups, and inverse Clifford semigroups, respectively, of which the latter three are full subcategories of CS.

Definition. A net $\left\{x_{\alpha}\right\}_{\alpha \in \Gamma}$ in a semilattice $X$ is said to be increasing if whenever $\alpha \leqslant \beta$ in $\Gamma$ we have $x_{\alpha} \leqslant x_{\beta}$ in $X$. We note here that if $X$ is a compact semilattice, then any increasing net $\left\{x_{\alpha}\right\}_{\alpha \in \Gamma}$ in $X$ converges to its supremum 
relative to the semilattice ordering (see Lemma 1.2 of [1]).

Definition. An inverse system $\left\{S_{\alpha} ; \varphi_{\beta}^{\alpha} ; \Gamma\right\}$ in a category $K$ consists of a directed set $\Gamma$, objects $S_{\alpha}$ of $K$ for each $\alpha \in \Gamma$, and morphisms $\varphi_{\beta}^{\alpha}: S_{\alpha} \rightarrow S_{\beta}$ in $K$ for each pair $\alpha, \beta \in \Gamma$ with $\beta \leqslant \alpha$, such that if $\alpha \leqslant \beta \leqslant \gamma$ in $\Gamma$, then $\varphi_{\alpha}^{\beta} \circ \varphi_{\beta}^{\gamma}=\varphi_{\alpha}^{\gamma}$. Note that if $\left\{x_{\alpha}\right\}_{\alpha \in \Gamma}$ is an increasing net in a semilattice $X$ and if $F$ is a functor from $X$ into any category $K$, then $\left\{x_{\alpha} ; x_{\alpha} \rightarrow x_{\beta} ; \Gamma\right\}$ is an inverse system in $X$ and $\left\{F\left(x_{\alpha}\right) ; F_{x_{\beta}}^{x_{\alpha}} ; \Gamma\right\}$ is an inverse system in $K$.

DEFinition. Let $\left\{S_{\alpha} ; \varphi_{\beta}^{\alpha} ; \Gamma\right\}$ be an inverse system in a category $K$. An inverse limit $\left\{S ; \varphi_{\alpha} ; \Gamma\right\}$ of the system $\left\{S_{\alpha} ; \varphi_{\beta}^{\alpha} ; \Gamma\right\}$ consists of an object $S$ of $K$ and morphisms $\varphi_{\alpha}: S \rightarrow S_{\alpha}$ for each $\alpha$ in $\Gamma$, such that $\varphi_{\alpha}=\varphi_{\alpha}^{\beta} \varphi_{\beta}$ whenever $\alpha \leqslant \beta$ in $\Gamma$, and such that if $\left\{T ; \psi_{\beta} ; \Gamma\right\}$ is any other such system, then there exists a unique morphism $\psi: T \rightarrow S$ such that $\psi_{\beta}=\varphi_{\beta} \psi$ for each $\beta \in \Gamma$.

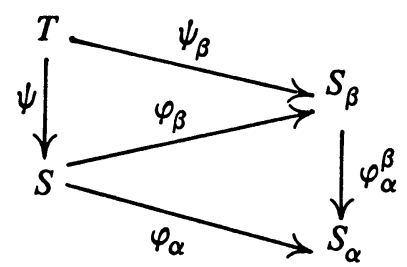

EXAMPLE. If $\left\{x_{\alpha}\right\}_{\alpha \in \Gamma}$ is an increasing net in a semilattice $X$ with supremum $x$, then $\left\{x ; x \rightarrow x_{\alpha} ; \Gamma\right\}$ is an inverse limit of the system $\left\{x_{\alpha} ; x_{\alpha} \rightarrow x_{\beta} ; \Gamma\right\}$.

Notation. Let $\left\{S_{\alpha} ; \varphi_{\beta}^{\alpha} ; \Gamma\right\}$ be an inverse system in the category of sets and functions. Let $\Pi_{\alpha \in \Gamma} S_{\alpha}$ denote the Cartesian product of the sets $S_{\alpha}$. We denote the subset $\left\{\left(s_{\alpha}\right)_{\alpha \in \Gamma}: \varphi_{\beta}^{\alpha}\left(s_{\alpha}\right)=s_{\beta}\right.$ whenever $\left.\beta \leqslant \alpha\right\}$ of $\Pi_{\alpha \in \Gamma} S_{\alpha}$ by $\stackrel{\lim }{\longleftarrow}\left\{S_{\alpha} ; \varphi_{\beta}^{\alpha} ; \Gamma\right\}$. For the sake of brevity, we shall use the notation $\lim _{\leftarrow} S_{\alpha}$ when

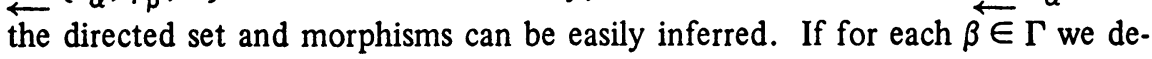
note by $\varphi_{\beta}$ the restriction to $\lim _{\longleftarrow} S_{\alpha}$ of the $\beta$ th projection $\pi_{\beta}: \Pi_{\alpha \in \Gamma} S_{\alpha} \rightarrow S_{\beta}$, then $\left\{\lim S_{\alpha} ; \varphi_{\alpha} ; \Gamma\right\}$ is an inverse limit of the system $\left\{S_{\alpha} ; \varphi_{\beta}^{\alpha} ; \Gamma\right\}$ provided the set $\lim _{\longleftarrow} S_{\alpha}$ is nonempty. Observe that if $\left\{S_{\alpha} ; \varphi_{\beta}^{\alpha} ; \Gamma\right\}$ is an inverse system in the category $C$ or the category CS, then $\lim S_{\alpha}$ still makes sense and $\left\{\lim S_{\alpha} ; \varphi_{\alpha} ; \Gamma\right\}$ is an inverse limit of $\left\{S_{\alpha} ; \varphi_{\beta}^{\alpha} ; \Gamma\right\}$ in that category.

Definition. Let $F$ be a functor from a category $K$ to a category $L$. Then we say that $F$ is inverse limit preserving if whenever $\left\{S ; \varphi_{\alpha} ; \Gamma\right\}$ is an inverse limit of the system $\left\{S_{\alpha} ; \varphi_{\beta}^{\alpha} ; \Gamma\right\}$ in $K$ then $\left\{F(S) ; F\left(\varphi_{\alpha}\right) ; \Gamma\right\}$ is an inverse limit of the system $\left\{F\left(S_{\alpha}\right) ; F\left(\varphi_{\beta}^{\alpha}\right) ; \Gamma\right\}$ in $L$. Thus a functor $F$ from a compact semilattice $X$ into a category $K$ is inverse limit preserving if and only if for each increasing net $\left\{x_{\alpha}\right\}_{\alpha \in \Gamma}$ in $X$ the system $\left\{F(x) ; F_{x_{\beta}}^{x_{\alpha}} ; \Gamma\right\}$ (where $x$ is the supremum of $\left\{x_{\alpha}: \alpha \in \Gamma\right\}$ ) is an inverse limit in $K$ of the system $\left\{F\left(x_{\alpha}\right) ; F_{x_{\beta}}^{x_{\alpha}} ; \Gamma\right\}$.

We omit the proof of the following lemma, which is rather straightforward 
and depends on the uniqueness (up to homeomorphism or isomorphism) of inverse limits in the categories concerned.

LEMMA 1. Let $F$ be a functor from a compact semilattice $X$ into the category $C$ (CS). Then $F$ is inverse limit preserving if and only if for each increasing net $\left\{x_{\alpha}\right\}_{\alpha \in \Gamma}$ in $X$ and supremum $x$ of that net the map $\varphi_{x}: F(x) \rightarrow \lim F\left(x_{\alpha}\right)$ de-

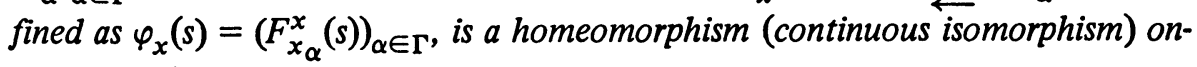
to $\lim _{\longleftarrow} F\left(x_{\alpha}\right)$.

\section{The construction theorems.}

DEFinition. Let $F$ be a functor from a semilattice $X$ into the category CS. We define the algebraic semigroup $S(F)$ to be the set $\bigcup_{x \in X}\{x\} \times F(x)$ with multiplication given by $(x, s)(y, t)=\left(x y, F_{x y}^{x}(s) F_{x y}^{y}(t)\right)$. That $S(F)$ is indeed a semigroup is an easy exercise. For more general algebraic constructions of this nature, see [8].

Consider now an inverse limit preserving functor $F$ from a compact semilattice $X$ into the category $G$ of compact groups. A. H. Clifford [3] showed that the algebraic semigroup $S(F)$ is an inverse semigroup which is the union of its subgroups, i.e., an inverse Clifford semigroup, and that any such semigroup could be thus constructed. In 1970, Bowman [1] described a topology on $S(F)$ for the case where the semilattice $X$ had a neighborhood basis of subsemilattices at each point. Relative to this topology $S(F)$ was a compact semigroup, and any compact inverse Clifford semigroup whose semilattice of idempotents had a Lawson basis for its topology could be thus constructed. Theorem 1 below gives a compact topology on $S(F)$ for the general case which is the "right" topology whenever that makes sense. That is, whenever $S(F)$ admits a compact semigroup topology relative to which the groups $\{x\} \times F(x)$ inherit their own topologies, then that topology is the topology given below. Before we proceed with our description of that topology, we shall need the following technique, which was used by J. D. Lawson in [6].

LEMmA 2. Let $X$ be a compact semilattice, and $x$ an element of $X$. Let $\Lambda$ be the collection of all sequences $\left\{U_{n}\right\}_{n=1}^{\infty}$ of open sets in $X$ such that for each $n$, $x \in U_{n+1} \subseteq \bar{U}_{n+1}^{2} \subseteq U_{n}$. Then $\Lambda$ is directed by $\left\{U_{n}\right\}_{n=1}^{\infty}<\left\{U_{n}^{\prime}\right\}_{n=1}^{\infty}$ if and only if $U_{n} \supseteq U_{n}^{\prime}$ for each $n$. Moreover, for each $\lambda=\left\{U_{n}\right\}_{n=1}^{\infty}$ in $\Lambda$ the intersection $\bigcap_{n=1}^{\infty} U_{n}$ is a compact subsemilattice of $X$ with zero $y_{\lambda}$, and $\left\{y_{\lambda}\right\}_{\lambda \in \Lambda}$ is an increasing net in $X$ with supremum $x$.

Definition. Let $F: X \rightarrow C$ be an inverse limit preserving functor from the compact semilattice $X$ into the category $C$. For each open subset $U$ of $X$, each element $u \in U$, and each open subset $V$ of $F(u)$, we define 


$$
W_{F}(U,(u, V))=\bigcup_{x \in U ; x \geqslant u}\{x\} \times\left(F_{u}^{x}\right)^{-1}(V) .
$$

When no confusion can result from our doing so we shall drop the subscript $F$.

Theorem 1. Let $X$ be a compact semilattice, and let $F: X \rightarrow C$ be an inverse limit preserving functor from $X$ into $C$. Let $S=\bigcup_{x \in X}\{x\} \times F(x)$, and let $T$ be the collection of subsets of $S$ defined as follows. A subset $O$ of $S$ is in $T$ if and only if for each element $(x, s)$ of $O$ there exists an open subset $U$ of $X$ such that

(1) $x \in U$; and

(2) if $y \in U$ and $y \leqslant x$, then there exists an open subset $V$ of $F(y)$ such that

(a) $(x, s) \in W(U,(y, V)) \subseteq 0$, and

(b) if $(z, t) \in W(U,(y, V))$ then for any $w \in U$ with $w \leqslant z$ there exists an open subset $W$ of $F(w)$ such that $(z, t) \in W(U,(w, W)) \subseteq 0$.

Then $T$ is a compact topology on $S(F)$.

Proof. That $T$ is a topology on $S(F)$ is a tedious but straightforward proof. We proceed to show that $T$ is a compact topology.

By the Axiom of Choice, there exists a function $C: X \rightarrow \bigcup_{x \in X} F(x)$ such that $C(x) \in F(x)$, for all $x \in X$. Let us fix such a function $C$ for later use.

Let $\left\{\left(x_{\alpha}, s_{\alpha}\right)\right\}_{\alpha \in \Gamma}$ be a net in $S$. Then the net $\left\{x_{\alpha}\right\}_{\alpha \in \Gamma}$ must cluster to some point $x \in X$. Suppose for contradiction that the net $\left\{\left(x_{\alpha}, s_{\alpha}\right)\right\}_{\alpha \in \Gamma}$ clusters to no point of $\{x\} \times F(x)$. Then for each element $t$ of $F(x)$, there exists $0_{t} \in T$ such that $(x, t) \in O_{t}$ and $\left\{\left(x_{\alpha}, s_{\alpha}\right)\right\}_{\alpha \in \Gamma}$ is eventually outside $O_{t}$. Now for each $t$, let $U_{t}$ be an open subset of $X$ and $V_{t}$ an open subset of $F(x)$ such that $(x, t) \in$ $W\left(U_{t},\left(x, V_{t}\right)\right) \subseteq 0_{t}$. Then $t \in V_{t}$. Hence the collection $\left\{V_{t}\right\}_{t \in F(x)}$ is an open covering of $F(x)$. Since $F(x)$ is compact, there exists a finite collection $\left\{t_{1}, t_{2}\right.$, $\left.\ldots, t_{n}\right\}$ of elements of $F(x)$ such that $F(x) \subseteq \bigcup_{i=1}^{n} V_{t_{i}}$. Hence $\{x\} \times F(x) \subseteq$ $\bigcup_{i=1}^{n} O_{t_{i}}$. But the net $\left\{\left(x_{\alpha}, s_{\alpha}\right)\right\}_{\alpha \in \Gamma}$ is eventually outside $O_{t_{i}}$, for $i=1,2, \ldots, n$. Hence $\left\{\left(x_{\alpha}, s_{\alpha}\right)\right\}_{\alpha \in \Gamma}$ is eventually outside $0=\bigcup_{i=1}^{n} O_{t_{i}}$. With no loss of generality, we assume the net is entirely outside 0 .

Now let $\left\{y_{\lambda}\right\}_{\lambda \in \Lambda}$ be the net in $X$ associated with the point $x \in X$, as described in Lemma 2. Fix $\lambda=\left\{U_{n}\right\}_{n=1}^{\infty}$ in $\Lambda$. We claim that there exists a sequence $\{\alpha(n)\}_{n=1}^{\infty}$ from $\Gamma$ such that $x_{\alpha(n)} x_{\alpha(n+1)} \cdots x_{\alpha(n+m)} \in U_{n}$, for all $n$ and $m$. Such a sequence may be chosen recursively, but we shall merely go over the first few steps. Firstly, the nets $\left\{x_{\alpha}\right\}_{\alpha \in \Gamma}$ and $\left\{x_{\alpha} x\right\}_{\alpha \in \Gamma}$ both converge in $X$ to $x$, so there exists $\alpha(1) \in \Gamma$ such that both $x_{\alpha(1)}$ and $x_{\alpha(1)} x$ are in $U_{1}$. By continuity there exists an open neighborhood $V_{1}$ of $x$ in $X$ such that $x_{\alpha(1)} V_{1}$ $\subseteq U_{1}$. Now we choose $\alpha(2)$ in $\Gamma$ such that both $x_{\alpha(2)}$ and $x_{\alpha(2)} x$ are in $U_{2} \cap$ $V_{1}$. Note that we have $x_{\alpha(1)} x_{\alpha(2)} \in x_{\alpha(1)} V_{1} \subseteq U_{1}$. To proceed to the next 
step, we note that $x_{\alpha(2)} x \in U_{2}$ and that $x_{\alpha(1)} x_{\alpha(2)} x \in x_{\alpha(1)} V_{1} \subseteq U_{1}$, so that by continuity of multiplication we obtain an open neighborhood $V_{2}$ of $x$ such that $x_{\alpha(2)} V_{2} \subseteq U_{2}$ and $x_{\alpha(1)} x_{\alpha(2)} V_{2} \subseteq U_{1}$. We then proceed to choose $\alpha(3)$ in $\Gamma$ so that both $x_{\alpha(3)}$ and $x_{\alpha(3)} x$ are in $U_{3} \cap V_{2}$. It is clear that this is a welldefined recursive process, by means of which we obtain the desired sequence $\{\alpha(n)\}_{n=1}^{\infty}$. Now for each positive integer $n$, the sequence

$$
\left\{x_{\alpha(n)} x_{\alpha(n+1)} \cdots x_{\alpha(n+m)}\right\}_{m=1}^{\infty}
$$

is a decreasing sequence in $X$ and therefore converges to its infimum, which we shall denote by $z_{n}$. But for positive integers $m, n$ we must have

$$
x_{\alpha(n)} x_{\alpha(n+1)} \cdots x_{\alpha(n+m)} \leqslant x_{\alpha(n+1)} x_{\alpha(n+2)} \cdots x_{\alpha(n+m)} .
$$

Hence $z_{n} \leqslant z_{n+1}$ for each positive integer $n$, since $\leqslant$ is a closed partial order on $X$. So the sequence $\left\{z_{n}\right\}_{n=1}^{\infty}$ is an increasing sequence and must therefore converge to its supremum $z_{\lambda}$. Note that $z_{n} \in \bar{U}_{n}$ for all $n$, so that $z_{\lambda} \in \bigcap_{n=1}^{\infty} U_{n}$ and hence $y_{\lambda} \leqslant z_{\lambda}$. Let $P_{\lambda}$ be the product space $\Pi_{n=1}^{\infty} F\left(z_{n}\right)$, and define a sequence $\left\{p_{n}\right\}_{n=1}^{\infty}$ in $P_{\lambda}$ as follows: for each positive integer $m$, the $m$ th coordinate of $p_{n}$ is given by

$$
\begin{aligned}
\pi_{m}\left(p_{n}\right) & =F_{z_{m}}^{x_{\alpha(n)}}\left(s_{\alpha(n)}\right), & & \text { if } m \leqslant n ; \\
& =C\left(z_{m}\right), & & \text { otherwise. }
\end{aligned}
$$

Let $p_{\lambda}$ be a cluster point in $P_{\lambda}$ of the sequence $\left\{p_{n}\right\}_{n=1}^{\infty}$. Notice that for all positive integers $i$ and $j$ with $i \leqslant j$ we must have $F_{z i}^{z j}\left(\pi_{j}\left(p_{\lambda}\right)\right)=\pi_{i}\left(p_{\lambda}\right)$, since for $m \geqslant j$ we have $F_{z_{i}}^{z_{j}}\left(\pi_{j}\left(p_{m}\right)\right)=\pi_{i}\left(p_{m}\right)$. Hence $p_{\lambda} \in \lim F\left(z_{n}\right)$. Since $F$ is inverse limit preserving, we know by Lemma 1 that the map $\varphi: F\left(z_{\lambda}\right) \rightarrow \lim F\left(z_{n}\right)$ defined as $\varphi(s)=\left(F_{z_{n}}^{z \lambda}(s)\right)_{n=1}^{\infty}$ is onto, so that there exists an element $t_{\lambda}$ of $F\left(z_{\lambda}\right)$ such that $F_{z_{n}}^{z}\left(t_{\lambda}\right)=\pi_{n}\left(p_{\lambda}\right)$, for all $n$.

Now since the sequences $\{\alpha(n)\}_{n=1}^{\infty},\left\{z_{n}\right\}_{n=1}^{\infty}$, and $\left\{p_{n}\right\}_{n=1}^{\infty}$ are dependent on $\lambda$, let us relabel them as $\alpha(n)=\alpha(\lambda, n), z_{n}=z_{\lambda n}$, and $p_{n}=p_{\lambda n}$.

Consider the product space $P=\Pi_{\lambda \in \Lambda} F\left(y_{\lambda}\right)$, and define the net $\left\{q_{\lambda}\right\}_{\lambda \in \Lambda}$ in $P$ as follows: for each $\gamma \in \Lambda$,

$$
\begin{aligned}
\pi_{\gamma}\left(q_{\lambda}\right) & =F_{y_{\gamma}}^{2}\left(t_{\lambda}\right), & & \text { if } \gamma \leqslant \lambda ; \\
& =C\left(y_{\gamma}\right), & & \text { otherwise. }
\end{aligned}
$$

Let $q$ be a cluster point in $P$ of the net $\left\{q_{\lambda}\right\}_{\lambda \in \Lambda}$. Then for fixed $\gamma, \delta \in \Lambda$ with $\gamma \leqslant \delta$ we have $F_{y_{\gamma}}^{y_{\delta}}\left(q_{\lambda}\right)=\pi_{\gamma}\left(q_{\lambda}\right)$ for all $\lambda \geqslant \delta$. Thus $q \in \lim F\left(y_{\lambda}\right)$. Since $F$ is inverse limit preserving, we can choose an element $u$ of $F(x)$ such that $F_{y_{\gamma}}^{x}(u)=$ $\pi_{\boldsymbol{\gamma}}(q)$ for each $\boldsymbol{\gamma} \in \Lambda$.

Now $(x, u) \in 0$, so there exists an open subset $U$ of $X$ such that $(x, s)=$ $(x, u)$ satisfies conditions (1) and (2) of the statement of the theorem. Choose 
$\lambda=\left\{U_{n}\right\}_{n=1}^{\infty}$ in $\Lambda$ such that $\bar{U}_{1} \subseteq U$. Since $y_{\lambda} \in \bigcap_{n=1}^{\infty} U_{n} \subseteq U_{1} \subseteq U$ and $y_{\lambda} \leqslant x$, there exists in $F\left(y_{\lambda}\right)$ an open neighborhood $V$ of $F_{y_{\lambda}}^{x}(u)$ such that if $y=y_{\lambda}$ and $s=u$ then (2a) and (2b) are satisfied. Now $F_{y_{\lambda}}^{x}(u)=\pi_{\lambda}(q)$, so $\pi_{\lambda}(q) \in V$.

Since the net $\left\{\pi_{\lambda}\left(q_{\gamma}\right)\right\}_{\gamma \in \Lambda}$ clusters to $\pi_{\lambda}(q)$, there exists $\delta \in \Lambda$ such that $\delta \geqslant \lambda$ and $\pi_{\lambda}\left(q_{\delta}\right) \in V$. So we obtain from $(\dagger)$ that $F_{y_{\lambda}}^{z}\left(t_{\delta}\right) \in V$. But since $\delta \geqslant \lambda$, $z_{\delta} \in U$. Hence $\left(z_{\delta}, t_{\delta}\right) \in W\left(U,\left(y_{\lambda}, V\right)\right)$. Note that $z_{\delta 1}$ is also in $U$, since if $\delta=\left\{U_{\delta n}\right\}_{n=1}^{\infty}$ then $z_{\delta 1} \in \bar{U}_{\delta 1} \subseteq \bar{U}_{1} \subseteq U$. Since $z_{\delta 1} \leqslant z_{\delta}$, by (2b) there exists an open subset $W$ of $F\left(z_{\delta 1}\right)$ such that $\left(z_{\delta}, t_{\delta}\right) \in W\left(U,\left(z_{\delta 1}, W\right) \subseteq 0\right.$. Hence $F_{z_{\delta}}^{z_{\delta}}\left(t_{\delta}\right) \in W$. But $F_{z_{\delta 1}}^{z_{\delta}}\left(t_{\delta}\right)=\pi_{1}\left(p_{\delta}\right)$, so $\pi_{1}\left(p_{\delta}\right) \in W$. Since the sequence $\left\{\pi_{1}\left(p_{\delta n}\right)\right\}_{n=1}^{\infty}$ clusters to $\pi_{1}\left(p_{\delta}\right)$, we must have $\pi_{1}\left(p_{\delta n}\right) \in W$ for some $n$. So by

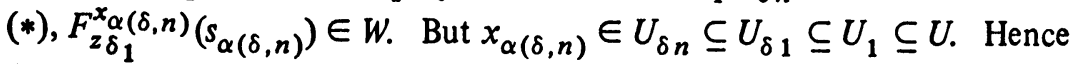
$\left(x_{\alpha(\delta, n)}, s_{\alpha(\delta, n)}\right) \in W\left(U,\left(z_{\delta 1}, W\right)\right) \subseteq 0$. But this is a contradiction to our assumption that the net $\left\{\left(x_{\alpha}, s_{\alpha}\right)\right\}_{\alpha \in \Gamma}$ lay entirely outside 0 . So the net $\left\{\left(x_{\alpha}, s_{\alpha}\right)\right\}_{\alpha \in \Gamma}$ does cluster to some point of $\{x\} \times F(x)$, and we conclude that the topology $T$ is compact.

Whether or not the above topology is Hausdorff in all cases is not clear. Any counterexample would have to be constructed from a non-Lawson semilattice, in view of Bowman's work and Corollary 4.2 of this paper, (for examples of non-Lawson semilattices, see [7]) and any direct attempts at a proof are hampered by the lack of a good description of a basis at any particular point of $S$. It should be mentioned here that any attempts at a proof of Hausdorffness would seem to need something like Lemma 3 of $\S 4$. Theorem 3 emphasizes the importance of the Hausdorffness question.

The following theorem demonstrates a method for constructing continuous maps between the spaces constructed in Theorem 1 .

THEOREM 2. Let each of $X$ and $Y$ be a compact semilattice, and let $F$ : $X \rightarrow C$ and $G: Y \rightarrow C$ be inverse limit preserving functors; let $S=\bigcup_{x \in X}\{x\} \times$ $F(x)$ and $S^{\prime}=\bigcup_{y \in Y}\{y\} \times G(y)$. Then any continuous homomorphism $\epsilon: X \rightarrow Y$ can be considered a functor, so that the composition $G \epsilon$ is a functor from $X$ into C. Let $\omega$ be a natural transformation from $F$ to $G \epsilon$. Then the function $f: S \rightarrow S^{\prime}$ defined as $f((x, s))=\left(\epsilon(x), \omega_{x}(s)\right)$ is a continuous map relative to the topologies on $S$ and $S^{\prime}$ given by Theorem 1 .

Proof. We first note that if $U^{\prime}$ is any open subset of $Y, U=\epsilon^{-1}\left(U^{\prime}\right)$, $y \in U, y^{\prime}=\epsilon(y), V^{\prime}$ an open subset of $G\left(y^{\prime}\right)$, and $V=\omega_{y}^{-1}\left(V^{\prime}\right)$, then

$$
f\left(w_{F}(U,(y, V))\right) \subseteq w_{G}\left(U^{\prime},\left(y^{\prime}, V^{\prime}\right)\right) .
$$

For if $(x, s) \in W_{F}(U,(y, V))$, then $x \in U$ and $y \leqslant x$, and $F_{y}^{x}(s) \in V$, which implies that $\epsilon(x) \in U^{\prime}, y^{\prime}=\epsilon(y) \leqslant \epsilon(x)$, and $\omega_{y} F_{y}^{x}(s) \in V^{\prime}$. Since $\omega$ is a natural transformation from $F$ to $G \epsilon$, we have $\omega_{y} F_{y}^{x}=G_{\epsilon(y)}^{\epsilon(x)} \omega_{x}$, so that $G_{y}^{\epsilon(x)}\left(\omega_{x}(s)\right)$ $\in V^{\prime}$. Hence $f((x, s))=\left(\epsilon(x), \omega_{x}(s)\right) \in W_{G}\left(U^{\prime},\left(y^{\prime}, V^{\prime}\right)\right)$. 
Now let $O^{\prime}$ be an open subset of $S^{\prime}$. We wish to show that $f^{-1}\left(O^{\prime}\right)=0$ is open in $S$. So let $(x, s) \in 0$, say $f((x, s))=\left(x^{\prime}, s^{\prime}\right) \in 0^{\prime}$. Then we choose an open neighborhood $U^{\prime}$ of $x^{\prime}$ in $Y$ which satisfies (1) and (2) of Theorem 1 relative to $\left(x^{\prime}, s^{\prime}\right)$ and $O^{\prime}$. Let $U=\epsilon^{-1}\left(U^{\prime}\right)$. Then if $y \in U$ and $y \leqslant x$, we have $y^{\prime}=\epsilon(y) \leqslant \epsilon(x)=x^{\prime}$, so there exists an open subset $V^{\prime}$ of $G \epsilon(z)$ such that (2a) and (2b) are satisfied. Let $V=\omega_{y}^{-1}\left(V^{\prime}\right)$. By the preceding paragraph, $W_{F}(U,(y, V)) \subseteq f^{-1}\left(W_{G}\left(U^{\prime},\left(y^{\prime}, V^{\prime}\right)\right)\right) \subseteq f^{-1}(0)=0^{\prime}$. Also, we have $x \in U$ and $y \leqslant x$, and since $\omega_{y} F_{y}^{x}(s)=G_{y^{\prime}}^{x^{\prime}} \omega_{x}(s)=G_{y^{\prime}}^{x^{\prime}}\left(s^{\prime}\right) \in V^{\prime}$, we have $F_{y}^{x}(s) \in$ $\omega_{y}^{-1}\left(V^{\prime}\right)=V$. So $(x, s) \in W_{F}(U,(y, V)) \subseteq 0$.

Now suppose $(z, t) \in W(U,(y, V))$, and suppose $w \in U$ with $w \leqslant z$. Then if $\epsilon(z)=z^{\prime}$ and $\omega_{z}(t)=t^{\prime}$, we have $\left(z^{\prime}, t^{\prime}\right) \in W_{G}\left(U^{\prime},\left(y^{\prime}, V^{\prime}\right)\right)$, and $w^{\prime}=\epsilon(w)$ is a point of $U^{\prime}$ below $z^{\prime}$ in $Y$, so that there exists an open subset $W^{\prime}$ of $G\left(w^{\prime}\right)$ such that $\left(z^{\prime}, t^{\prime}\right) \in W_{G}\left(U^{\prime},\left(w^{\prime}, W^{\prime}\right)\right) \subseteq 0^{\prime}$. We now argue exactly as before that if $W=\omega_{w}^{-1}\left(W^{\prime}\right)$ then $(z, t) \in W_{F}(U,(w, W) \subseteq 0$. We conclude that $O$ is open and therefore that $f$ is continuous.

Note the use of Theorem 2 in the proof of the following theorem.

THEOREM 3. Let $X$ be a compact semilattice, and let $F: X \rightarrow C S$ be an inverse limit preserving functor into the category CS of compact semigroups. Let $S(F)$ have the topology as described in Theorem 1. Then multiplication is separately continuous on $S(F)$, and if $S(F)$ is Hausdorff, then $S(F)$ is a compact semigroup.

Proof. We first show that multiplication is separately continuous on $S(F)$. Let $(x, s) \in S(F)$, and let $\lambda: S(F) \rightarrow S(F)$ be left translation by $(x, s)$. Then the left translation $\lambda_{x}: X \rightarrow X$ defined by $\lambda_{x}(y)=x y$ is a continuous homomorphism on $X$. Let $\omega: F \rightarrow F \lambda_{x}$ be defined by $\omega_{y}(t)=F_{x y}^{x}(s) F_{x y}^{y}(t)$. We observe that $\omega$ is a natural transformation since the following diagram is commutative for all $y$ and $z$ with $z \leqslant y$.

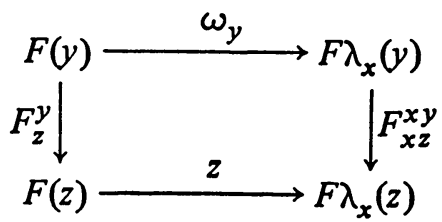

Since $\lambda(y, t)=\left(\lambda_{x}(y), \omega_{y}(t)\right)$ for all $(y, t) \in S(F)$, we conclude by Theorem 2 that $\lambda$ is continuous. So all left translations are continuous. Similarly all right translations are continuous.

The only thing to be shown now is that if $S(F)$ is Hausdorff, then the multiplication $m: S(F) \times S(F) \rightarrow S(F)$ is continuous. We shall prove this fact by showing that there exists an inverse-limit-preserving functor $G$ from the compact semilattice $X \times X$ into the category CS such that $S(F)$ as a topological space is homeomorphic to $S(F) \times S(F)$ and such that the map $n: S(G) \rightarrow S(F)$ induced by the following diagram is continuous. 


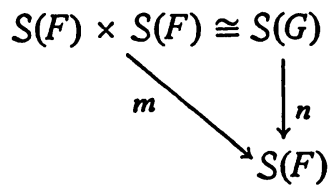

Let $G: X \times X \rightarrow C S$ be defined by $G((x, y))=F(x) \times F(y)$ and $G_{(z, w)}^{(x, y)}((s, t))=\left(F_{z}^{x}(s), F_{w}^{y}(t)\right)$ whenever $z \leqslant x$ and $w \leqslant y$. It is a straightforward exercise to show that $G$ is an inverse limit preserving functor. It is also clear that the map $\alpha: S(G) \rightarrow S(F) \times S(F)$ defined as $\alpha((x, y),(s, t))=((x, s),(y, t))$ is a bijection. We proceed to show that $\alpha$ is continuous. For this it suffices to show that $\pi_{1} \alpha$ and $\pi_{2} \alpha$ are continuous. But note that $\pi_{1}: X \times X \rightarrow X$ is a continuous homomorphism and that $\omega: G \rightarrow F \pi_{1}$ defined as $\omega_{(x, y)}((s, t))=s$ is a natural transformation, with $\pi_{1} \alpha((x, y),(s, t))=\left(\pi_{1}(x, y), \omega_{(x, y)}(s, t)\right)$, so that $\pi_{1} \alpha$ is continuous by Theorem 2. Similarly, $\pi_{2} \alpha$ is continuous. So $\alpha$ is a topological isomorphism between $S(G)$ and $S(F) \times S(F)$.

We show that $n: S(G) \rightarrow S(F)$ defined as

$$
n((x, y),(s, t))=\left(x y, F_{x y}^{x}(s) F_{x y}^{y}(t)\right)
$$

is continuous in the same way. For if $m^{\prime}: X \times X \rightarrow X$ is the multiplication on the semilattice $X$, then $m^{\prime}$ is a continuous homomorphism, since it is commutative. We now define a natural transformation $\mu: G \rightarrow F m^{\prime}$ as $\mu_{(x, y)}(s, t)=F_{x y}^{x}(s) F_{x y}^{y}(t)$. (The reader should verify that the following diagram is indeed commutative whenever $(z, w) \leqslant(x, y)$.)

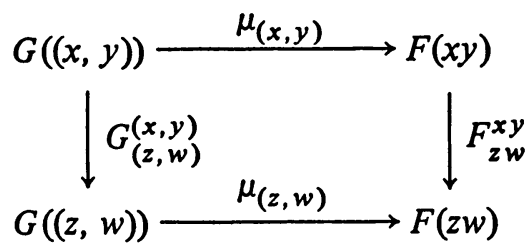

Finally, we note that for each $((x, y),(s, t)) \in S(G), n((x, y),(s, t))=\left(m^{\prime}(x, y)\right.$, $\left.\mu_{(x, y)}(s, t)\right)$, so that $n$ is continuous by Theorem 2. Hence $m$ is continuous and $S(F)$ is a compact semigroup.

3. The category $\mathrm{Cl}$. The following theorem describes the process by which one can reconstruct an object $S$ of $C l$ from its semilattice of idempotents, its maximal subgroups $H(e)$, and the maps $s \rightarrow$ se which carry $H(f)$ into $H(e)$ whenever $e$ and $f$ are idempotents with $e \leqslant f$.

THEOREM 4. Let $S$ be a compact inverse Clifford semigroup with idempotents $X$. Then $X$ is a compact semilattice, and the functor $F: X \rightarrow G$ defined by $F(x)=H(x)$ for each $x \in X$ and $F_{y}^{x}(s)=y s$ for each $x \in X, s \in H(x)$, and $y \leqslant x$, is inverse limit preserving. Furthermore, $S(F)$ is a compact semigroup with the 
topology of Theorem 1, and $S(F)$ and $S$ are topologically isomorphic under the correspondence $(x, s) \rightarrow s$.

Proof. That $X$ is a compact semilattice is well known. That $F: X \rightarrow G$ is indeed a functor was done by Clifford [3], and the fact that it is inverse limit preserving is due to Hunter [5]. It is clear that the map $(x, s) \rightarrow s$ is an algebraic isomorphism of $S(F)$ onto $S$. So all that is left for us to show is the continuity of that map.

Let $\varphi: S(F) \rightarrow S$ be defined by $\varphi(x, s)=s$, and let $M$ be an open subset of $S$. Suppose for contradiction that $O=\varphi^{-1}(M)$ is not open in $S(F)$. Then for some point $(x, s)$ in 0 , it must be true that there is no open subset $U$ of $X$ which satisfies (1) and (2) of Theorem 1 . Let such a point $(x, s)$ in 0 be fixed.

Fix an open neighborhood $U$ of $x$ in $X$. Then $U$ does not satisfy condition (2), so there exists an element $y_{U}$ of $U$ such that $y_{U} \leqslant x$ and no open neighborhood $V$ of $F_{y_{U}}^{x}(s)$ in $F\left(y_{U}\right)$ will satisfy (2a) and (2b).

Fix an open neighborhood $V$ of $F_{y_{U}}^{x}(s)$ in $F\left(y_{U}\right)$. Then $V$ fails to satisfy either (2a) or (2b). But failure to satisfy (2a) implies failure to satisfy (2b), so without loss of generality $V$ fails to satisfy (2b). Thus there exists an element $\left(x_{(U, V)}, s_{(U, V)}\right)$ of $W\left(U,\left(y_{U}, V\right)\right)$ and an element $z_{(U, V)}$ of $U$ such that $z_{(U, V)}$ $\leqslant x_{(U, V)}$ and for any open neighborhood $W$ of $F_{z(U, V)}^{x}(U, V)\left(s_{(U, V)}\right)$ in $F\left(z_{(U, V)}\right)$ it is false that $W\left(U,\left(z_{(U, V)}, W\right) \subseteq 0\right.$. So for each such $W$, choose a point $\left(w_{(U, V, W)}, t_{(U, V, W)} \in W\left(U,\left(z_{(U, V)}, W\right)\right) \backslash 0\right.$.

Now let $\Lambda$ be the collection of all open neighborhoods $W$ of $F_{z}^{x}(U, V)(s(U, V)$ in $F\left(z_{(U, V)}\right)$. Then $\Lambda$ is directed by reverse inclusion. The net $\left\{t_{(U, V, W)}\right\}_{W \in \Lambda}$ is contained in $S \backslash M$, so it must cluster to a point $t_{(U, V)}$ in $S \backslash M$. Recall that $z_{(U, V)} t_{(U, V, W)}=F_{z(U, V)}^{w}(U, W)\left(t_{(U, V, W)}\right) \in W$ for each $W \in \Lambda$. We wish to use this fact to show that

$$
z_{(U, V)} t_{(U, V)}=z_{(U, V)} s_{(U, V)}
$$

Well, if $N$ is any open neighborhood of $z_{(U, V)} s_{(U, V)}$ in $S$, then $W=N \cap F\left(z_{(U, V)}\right)$ is an element of $\Lambda$, and if $W^{\prime} \in \Lambda$ with $W^{\prime} \subseteq W$ then $z_{(U, V)}{ }_{\left(U, V, W^{\prime}\right)} \in W^{\prime} \subseteq W$ $\subseteq N$, so the net $\left\{z_{(U, V)} t_{(U, V, W)}\right\}_{W \in \Lambda}$ converges in $S$ to $z_{(U, V)} s_{(U, V)}$. But by continuity of multiplication that net also clusters to $z_{(U, V)}{ }^{t}(U, V)$. Thus we have shown that (*) holds.

Now let us allow $V$ to vary. Let $\Sigma$ be the collection of all open neighborhoods $V$ of $F_{y_{U}}^{x}(s)$ in $F\left(y_{U}\right)$ directed by reverse inclusion. Consider the net $\left\{z_{(U, V)}, s_{(U, V)}, t_{(U, V)}\right\}_{V \in \Sigma}$ in $X \times S \times S$. It must cluster to some point $\left(z_{U}, s_{U}, t_{U}\right)$ in $X \times S \times S$. Note that the map $(x, s, t) \rightarrow(x s, x t)$ is continuous, so that $\left\{\left(z_{(U, V} v_{(U, V}, z_{(U, V}{ }^{t}(U, V)\right)\right\}_{V \in \Sigma}$ clusters at $\left(z_{U} s_{U}, z_{U} t_{U}\right)$. But by (*) that net is contained in $\Delta_{S}$. Hence $\left(z_{U} s_{U}, z_{U} t_{U}\right) \in \Delta_{S}$. That is, 


$$
z_{U} s_{U}=z_{U} t_{U}
$$

Note also that since $\left(x_{(U, V)}, s_{(U, V)}\right) \in W\left(U,\left(y_{U}, V\right)\right)$, we have $y_{U} s_{(U, V)} \in V$, for all $V \in \Sigma$. By an argument like that used in proving (*), we can show that

$$
y_{U} s_{U}=y_{U} s .
$$

Note that for all $V \in \Sigma, t_{(U, V)} \notin M$, so that $t_{U} \notin M$.

Finally, let $\Gamma$ be the collection of all open neighborhoods of $x$ in $X$, directed by reverse inclusion. Then the net $\left\{\left(y_{U}, z_{U}, s_{U}, t_{U}\right)\right\}_{U \in \Gamma}$ must cluster to a point $\left(y, z, s^{\prime}, t\right)$ in $X \times X \times S \times S$. Furthermore, since $\eta: S \rightarrow X$ taking an element $s$ of $S$ to the idempotent in $H(s)$ is continuous, and since $y_{U}, z_{U}, \eta\left(s_{U}\right)$, and $\eta\left(t_{U}\right)$ are all in $\bar{U}$ for each $U \in \Gamma$, we must have $y=z=\eta\left(s^{\prime}\right)=\eta(t)=x$. By $(\dagger)$, we have $z s^{\prime}=z t$, that is, $s^{\prime}=t$. By $(\nu)$, we have $y s^{\prime}=y s$, or $s^{\prime}=s$. Hence $s=t$. But $t_{U} \notin M$, for all $U$, so $t \notin M$. Thus $s \notin M$, which is a contradiction. So $O$ is open and $\varphi$ is continuous.

Hence $S(F)$ and $S$ are topologically isomorphic, and the proof is complete.

CoROllary 4.1. Let each of $S$ and $S^{\prime}$ be a compact inverse Clifford semigroup, and let $f: S \rightarrow S^{\prime}$ be an algebraic homomorphism. If $f$ is continuous from the idempotents $X$ of $S$ to the idempotents $Y$ of $S^{\prime}$ and on each of the subgroups of $S$, then $f$ is continuous on $S$.

Proof. Let $F: X \rightarrow G$ and $G: Y \rightarrow G$ be the maximal group functors, and let $\epsilon: X \rightarrow Y$ and $\omega_{x}: F(x) \rightarrow G \epsilon(x)$ be the restrictions of $f$, for each $x \in X$. Then $\omega$ is a natural transformation from $F$ to $G \epsilon$, so the map $f^{\prime}: S(F) \rightarrow S(G)$ defined by by $f^{\prime}(x, s)=\left(\epsilon(x), \omega_{x}(s)\right)$ is continuous. We obtain the result now by commutativity of the following diagram, where $\varphi: S(F) \rightarrow S$ and $\varphi^{\prime}: S(G) \rightarrow S^{\prime}$ are the topological isomorphisms given by Theorem 4 .

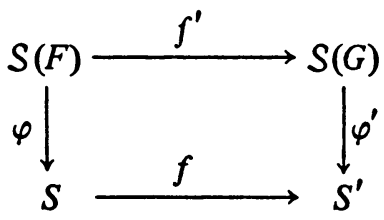

COROLlary 4.2. Let each of $S$ and $S^{\prime}$ be a compact inverse Clifford semigroup, and let $f: S \rightarrow S^{\prime}$ be an algebraic isomorphism of $S$ onto $S^{\prime}$. If the restriction of $f$ to each of the subgroups of $S$ is continuous, then $f$ is a topological isomorphism. Hence an inverse Clifford semigroup admits at most one compact semigroup topology which induces a preassigned topology on each of its subgroups.

Proof. If $\epsilon: X \rightarrow Y$ denotes the restriction of $f$ to the idempotents $X$ of $S$, then $\epsilon$ is continuous by Corollary 16 of [6]. Hence $f$ is continuous by Corollary 4.1 . 
The preceding theorems motivate the following definition, which generalizes Bowman's definition in [1]. The notation is patterned after that of Carruth and Clark in [2].

Definition. The category $F(\Omega ; G)$ is defined to be the category whose objects are inverse limit preserving functors $F: X \rightarrow G$ from compact semilattices $X$ into $G$, with morphisms given as follows. For objects $F: X \rightarrow G$ and $G: Y \rightarrow G$ of $F(\Omega ; G)$, a morphism from $F$ to $G$ is a pair $(\epsilon, \omega)$, where $\epsilon: X \rightarrow Y$ is a continuous homomorphism and $\omega: F \rightarrow G \epsilon$ is a natural transformation. The composition of two morphisms $(\epsilon, \omega): F \rightarrow G$ and $\left(\epsilon^{\prime}, \omega^{\prime}\right): G \rightarrow H$ is given by $\left(\epsilon^{\prime}, \omega^{\prime}\right) \circ(\epsilon, \omega)=$ $\left(\epsilon^{\prime} \circ \epsilon, \omega_{\epsilon}^{\prime} \circ \omega\right)$, where $\left(\omega_{\epsilon}^{\prime}\right)_{x}$ is $\omega_{\epsilon(x)}^{\prime}: G \epsilon(x) \rightarrow H \epsilon^{\prime} \epsilon(x)$, for each $x$ in $X$.

DEFinition. We define $F^{\prime}(\Omega ; G)$ to be the full subcategory of $F(\Omega ; G)$ whose objects $F$ satisfy the property that $S(F)$ is Hausdorff.

We now have the major components to a proof of the following theorem.

THEOREM 5. Cl is equivalent to $F^{\prime}(\Omega ; G)$.

OUTLINE OF PROOF. Let $K: C \ell \rightarrow F^{\prime}(\Omega ; G)$ be defined as follows. For each object $S$ of $C l$, with idempotents $X$, let $K(S)$ be the maximal group functor $F: X \rightarrow G$, and for each morphism $f: S \rightarrow S^{\prime}$ in $C \ell$, let $K(f)=(\epsilon, \omega)$ where $\epsilon$ and $\omega_{x}$ are restrictions of $f$, for each $x$ in $X$. Let $L: F^{\prime}(\Omega ; G) \rightarrow C l$ be such that $L(F)$ is the semigroup $S(F)$ with the topology of Theorem 1 , and

$$
L(\epsilon, \omega)(x, s)=\left(\epsilon(x), \omega_{x}(s)\right)
$$

for each morphism $(\epsilon, \omega): F \rightarrow G$ in $F^{\prime}(\Omega ; G)$ and each point $(x, s)$ in $S(F)$. One then checks that $K$ and $L$ are indeed functors, that $K \circ L$ is naturally equivalent to $1_{F^{\prime}(\Omega ; G)}$, and that $L \circ K$ is naturally equivalent to $1_{C \ell}$.

4. The first axiom semilattice case. Here we consider the case of compact inverse Clifford semigroups $S$ with idempotents $X$, where $X$ is first axiom. In this case the topology is described by the following theorem.

THEOREM 6. Let $X$ be a compact first axiom semilattice, and let $F: X \rightarrow$ $C$ be an inverse limit preserving functor. Let $S=\bigcup_{x \in X}\{x\} \times F(x)$, and let $T$ be the collection of subsets $O$ of $S$ which satisfy the following property. For each element $(x, s)$ of 0 , there exists an open subset $U$ of $X$ such that

(1') $x \in U$; and

(2') if $y \in U$ and $y \leqslant x$, then there exists an open subset $V$ of $F(y)$ such that $(x, s) \in W(U,(y, V)) \subseteq 0$.

Then $T$ is a compact topology on $S$.

Proof. That $T$ is a topology is a straightforward argument. To show that $T$ is compact, we proceed as in Theorem 1 to assume the negation and obtain a net $\left\{\left(x_{\alpha}, s_{\alpha}\right)\right\}_{\alpha \in \Gamma}$ in $S$, a point $x \in X$, and a set $0 \in T$ with $\{x\} \times F(x) \subseteq 0$, such 
that $x_{\alpha}$ clusters to $x$ but the net $\left\{\left(x_{\alpha}, s_{\alpha}\right)\right\}_{\alpha \in \Gamma}$ is entirely outside 0 . We then note that the net $\left\{y_{\lambda}\right\}$ given by Lemma 2 is eventually constant, i.e., there exists $\lambda$ such that $y_{\lambda}=z_{\lambda}=x$. Thus $t_{\lambda} \in F(x)$, so $\left(x, t_{\lambda}\right) \in 0$. Let $U$ satisfy ( $\left.1^{\prime}\right)$ and (2') above with $s=t_{\lambda}$. Then there exists $n$ such that $z_{\lambda n} \in U$ and $U_{\lambda n} \subseteq U$. Let $V$ be an open subset of $F\left(z_{\lambda n}\right)$ such that $\left(x, t_{\lambda}\right) \in W\left(U,\left(z_{n}, V\right)\right) \subseteq 0$. Then $\pi_{n}\left(p_{\lambda}\right)=F_{z \lambda n}^{x}\left(t_{\lambda}\right) \in V$, so there exists $m \geqslant n$ such that $\pi_{n}\left(p_{\lambda m}\right) \in V$. That is, $F_{z \lambda n}^{x_{\alpha}(\lambda, m)}\left(s_{\alpha(\lambda, m)}\right) \in V$. But $x_{\alpha(\lambda, m)} \in U_{\lambda m} \subseteq U_{\lambda n} \subseteq U$, so $\left(x_{\alpha(\lambda, m)}, s_{\alpha(\lambda, m)}\right)$ $\in W\left(U,\left(z_{n}, V\right)\right) \subseteq O$, a contradiction.

ReMARK. Let $X$ be a compact first axiom semilattice, and let $F: X \rightarrow C$ be an inverse limit preserving functor. Let $S=\bigcup_{x \in X}\{x\} \times F(x)$, and let $T_{1}$ and $T_{2}$ be the topologies on $S$ defined by Theorems 1 and 6, respectively. Clearly, then, $T_{1} \subseteq T_{2}$. Hence, whenever $T_{1}$ is Hausdorff, we will have $T_{1}=T_{2}$. So the topology on a compact inverse Clifford semigroup with first axiom semilattice of idempotents is given by Theorem 6 . We state this result as a corollary.

COROLlaRy 6. Let $S$ be a compact inverse Clifford semigroup with idempotents $X$, and suppose $X$ is first axiom. Let $F: X \rightarrow G$ be the maximal group functor. Then $S(F)$ is a compact semigroup when given the topology of Theorem 6 , and the map $(x, s) \rightarrow s$ of $S(F)$ onto $S$ is a topological isomorphism.

5. The Hausdorffness question-a partial result. The following lemma would seem to indicate an affirmative answer to the Hausdorffness question. We will use it as a tool to achieve a partial result along these lines in Theorem 7.

Lemma 3. Let $X$ be a compact semilattice, and let $F: X \rightarrow C$ be an inverse limit preserving functor. Then for any $x \in X$ and any pair of nonempty disjoint closed subsets $A$ and $B$ of $F(x)$, there exists a neighborhood $U$ of $x$ such that if $y \in U$ and $y \leqslant x$, then $F_{y}^{x}(A) \cap F_{y}^{x}(B)=\varnothing$.

Proof. Suppose no such neighborhood exists. Then every open set $U$ containing $x$ contains a $y_{U} \leqslant x$ such that

$$
F_{y_{U}}^{x}(A) \cap F_{y_{U}}^{x}(B) \neq \varnothing .
$$

Now let $\left\{y_{\lambda}\right\}_{\lambda \in \Lambda}$ be Lawson's net converging up to $x$, as described in Lemma 1.

Fix $\lambda \in \Lambda$, say $\lambda=\left\{U_{n}\right\}_{n=1}^{\infty}$. Let $y_{1} \in U_{1}$ such that $y_{1} \leqslant x$ and $F_{y_{1}}^{x}(A) \cap$ $F_{y_{1}}^{x}(B) \neq \varnothing$. Choose a neighborhood $V_{1}$ of $x$ such that $y_{1} V_{1} \subseteq U_{1}$. Let $W_{1}=$ $U_{1}$ and $W_{2}=V_{1} \cap U_{2}$. Then choose $y_{2} \in W_{2}$ such that $y_{2} \leqslant x$ and $F_{y_{2}}^{x}(A) \cap$ $F_{y_{2}}^{x}(B) \neq \varnothing$. Again choose an open neighborhood $V_{2}$ of $x$ such that $y_{2} V_{2} \subseteq W_{2}$. Then $y_{2} \in U_{2}$ and $y_{1} y_{2}=y_{1} y_{2} x \in y_{1} y_{2} V_{2} \subseteq y_{1} W_{2} \subseteq y_{1} V_{1} \subseteq U_{1}$. We can thus recursively define a sequence $\left\{y_{n}\right\}_{n=1}^{\infty}$ in $X$ such that for each positive integer $n$, we have 
(1) $y_{n} \leqslant x$ and $F_{y_{n}}^{x}(B) \neq \varnothing$, and

(2) the sequence $\left\{y_{n} y_{n+1} \cdots y_{n+m}\right\}_{m=0}^{\infty}$ is contained in $U_{n}$.

Now for each positive integer $n$, the sequence $\left\{y_{n} y_{n+1} \cdots y_{n+m}\right\}_{m=0}^{\infty}$ converges to its infimum $z_{n} \in \bar{U}_{n}$. Recall from the proof of Theorem 1 that the sequence $\left\{z_{n}\right\}_{n=1}^{\infty}$ is an increasing sequence and converges to its supremum $z_{\lambda}$, and that $y_{\lambda} \leqslant z_{\lambda}$. Now for each positive integer $n$, note that $F_{z_{n}}^{y_{n}}\left(F_{y_{n}}^{x}(A) \cap F_{y_{n}}^{x}(B)\right) \subseteq$ $F_{z_{n}}^{x}(A) \cap F_{z_{n}}^{x}(B)$, so that $F_{z_{n}}^{x}(A) \cap F_{z_{n}}^{x}(B) \neq \varnothing$. Hence for each $n$ we can choose $a_{n} \in A$ and $b_{n} \in B$ such that $F_{z_{n}}^{x}\left(a_{n}\right)=F_{z_{n}}^{x}\left(b_{n}\right)$. The sequence $\left\{\left(a_{n}, b_{n}\right)\right\}_{n=1}^{\infty}$ must cluster to some point $\left(a_{\lambda}, b_{\lambda}\right)$ of $A \times B$. We claim that $F_{y_{\lambda}}^{x}\left(a_{\lambda}\right)=F_{y_{\lambda}}^{x}\left(b_{\lambda}\right)$. For each positive integer $m$, the sequence

$$
\left\{\left(F_{z_{m}}^{x} \times F_{z_{m}}^{x}\right)\left(a_{n}, b_{n}\right)\right\}_{n \geqslant m}
$$

is contained in the diagonal $\Delta_{F\left(z_{m}\right)}$. Hence $\left(F_{z_{m}}^{x} \times F_{z_{m}}^{x}\right)\left(a_{\lambda}, b_{\lambda}\right) \in \Delta_{F\left(z_{m}\right)}$, since $F_{z_{m}}^{x}$ is continuous. So $F_{z_{m}}^{x}\left(a_{\lambda}\right)=F_{z_{m}}^{x}\left(b_{\lambda}\right)$, for all $m$. But since $F$ is inverse limit preserving, this means $F_{z_{\lambda}}^{x}\left(a_{\lambda}\right)=F_{z_{\lambda}}^{x}\left(b_{\lambda}\right)$, and consequently $F_{y_{\lambda}}^{x}\left(a_{\lambda}\right)=$ $F_{y_{\lambda}}^{x}\left(b_{\lambda}\right)$. Notice that we have found a net $\left\{\left(a_{\lambda}, b_{\lambda}\right)\right\}_{\lambda \in \Lambda}$ in $A \times B$ such that $F_{y_{\lambda}}^{x}\left(a_{\lambda}\right)=F_{y_{\lambda}}^{x}\left(b_{\lambda}\right)$, for each $\lambda \in \Lambda$. That net must cluster to some point $(a, b)$ $\in A \times B$, which must satisfy $F_{y_{\lambda}}^{x}(a)=F_{y_{\lambda}}^{x}(b)$ for each $\lambda \in \Lambda$. Since $F$ is inverse limit preserving, we have $a=b$. Thus we have reached a contradiction, and the theorem is proven.

Definition. $C_{0}$ is defined to be the subcategory of $C$ whose morphisms are open maps. $G_{0}$ and $C S_{0}$ are defined similarly.

THEOREM 7. Let $X$ be a compact semilattice, and let $F: X \rightarrow C_{0}$ be an inverse limit preserving functor. Let $S$ be the set $\bigcup_{x \in X}\{x\} \times F(x)$, and let $T$ be the topology on $S$ given by Theorem 1. Then $T$ is Hausdorff.

Proof. Let $U$ and $V$ be open subsets of $X$ with $V^{2} \subseteq U, v \in V$, and $W$ an open subset of $F(v)$. Define $B(U, V,(v, W))$ to be those elements $(x, s)$ of $S$ such that $x \in V$ and for which there exists $z \in U$ such that $z \leqslant x v$ and $F_{z}^{x}(s) \in$ $F_{z}^{v}(W)$. Then $B(U, V,(v, W))$ is in $T$ for all such $U, V, v$, and $W$.

Now let $(x, s)$ and $(y, t)$ be distinct elements of $S$. If $x \neq y$, then there exist disjoint open sets $U$ and $U^{\prime}$ about $x$ and $y$, respectively, in $X$. Let

$$
0=\bigcup_{x \in U}\{x\} \times F(x), \quad O^{\prime}=\bigcup_{x \in U}\{x\} \times F(x) .
$$

Then $O$ and $O^{\prime}$ are disjoint neighborhoods in $S$ about $(x, s)$ and $(y, t)$.

Suppose now that $x=y$ and $s \neq t$. Let $W$ and $W^{\prime}$ be neighborhoods in $F(x)$ about $s$ and $t$, respectively, such that $\bar{W} \cap \overline{W^{\prime}}=\varnothing$. Let $M$ be an open neighborhood in $X$ about $x$ such that whenever $y \in M$ and $y \leqslant x$, then $F_{y}^{x}(\bar{W}) \cap$ $F_{y}^{x}\left(\overline{W^{\prime}}\right)=\varnothing$. Let $U$ and $V$ be open subsets of $X$ such that $x \in V \subseteq V^{2} \subseteq U \subseteq$ $U^{2} \subseteq M$. Then $(x, s) \in B(U, V,(x, W)),(x, t) \in B\left(U, V,\left(x, W^{\prime}\right)\right)$, and 


$$
B\left(U, V,(x, W) \cap B\left(U, V,\left(x, W^{\prime}\right)\right)=\varnothing .\right.
$$

Thus $(x, s)$ and $(y, t)$ can be separated by sets in $T$, and the proof is complete.

Definition. Let $S$ be a compact inverse Clifford semigroup. We say that the multiplication on $S$ is full if for any two idempotents $x, y$ in $S$ with $x \leqslant y$ we have that $x H(y)$ is an open subset of $H(x)$. We then define the category $C \ell_{f}$ to be the full subcategory of $\mathrm{Cl}$ whose objects have full multiplications.

COROllary 7.1. Let $X$ be a compact semilattice, and $F: X \rightarrow C S_{0}$ an inverse limit preserving functor. Then $S(F)$ is a compact semigroup with the topology of Theorem 1; moreover, if the codomain of $F$ is $G_{0}$, then $S(F)$ is an object of $\mathrm{Cl}_{f}$.

COROLLARY 7.2. $C \ell_{f}$ is equivalent to the full subcategory $F\left(\Omega ; G_{0}\right)$ of $F(\Omega ; G)$ whose objects are functors $F: X \rightarrow G_{0}$ from compact semilattices $X$ into the category $G_{0}$.

\section{BIBLIOGRAPHY}

1. T. T. Bowman, A construction principle and compact Clifford semigroups, Semigroup Forum 2 (1971), 343-353.

2. J. H. Carruth and C. E. Clark, H-coextensions in the category of compact semigroups, Semigroup Forum 7 (1974), 164-179.

3. A. H. Clifford and G. B. Preston, The algebraic theory of semigroups. Vol. II, Math. Surveys, no. 7, Amer. Math. Soc., Providence, R. I., 1961. MR 24 \#A2627.

4. E. Hewitt and K. A. Ross, Abstract harmonic analysis. Vol. I: Structure of topolog. ical groups. Integration theory, group representations, Die Grundlehren der math. Wissenschaften, Band 115, Academic Press, New York; Springer-Verlag, Berlin, 1963. MR 28 \#158.

5. R. P. Hunter, Certain homomorphisms of compact connected semigroups, Duke Math. J. 28 (1961), 83-87. MR 23 \#A629.

6. J. D. Lawson, Intrinsic topologies in topological lattices and semilattices, Pacific J. Math. 44 (1973), 593-602. MR 47 \#6580.

7. - Lattices with no interval homomorphisms, Pacific J. Math. 32 (1970), 459-465. MR $41 \# 1019$.

8. J. E. Leech, Two papers: H-coextensions of monoids and The structure of a band of groups, Mem. Amer. Math. Soc. No. 157 (1975).

9. B. L. van der Waerden, Stetigkeitssätze für halbeinfache Liesche Gruppen, Math. Z. 36 (1933), 780-786.

DEPARTMENT OF MATHEMATICS, LOUISIANA STATE UNIVERSITY, BATON ROUGE, LOUISIANA 70803 